\title{
COORDINATED FAULT TOLERANT OPTIMAL CONTROL OF AUTONOMOUS AGENTS: GEOMETRY AND COMMUNICATIONS ARCHITECTURE*
}

\author{
RYAN O. ABEL ${ }^{\dagger}$, SOURA DASGUPTA ${ }^{\dagger}$, AND JON G. KUHL ${ }^{\dagger}$
}

\begin{abstract}
A one step ahead optimization based control of autonomous agents is proposed. Each agent is modeled as a double integrator. We only define a geometric topology for the agent formation, and by correctly choosing the cost function, show that our algorithm produces a communication topology mirroring the geometric topology. By providing some redundancy in the formation topology it is possible for the system to survive the loss of an agent. Other attractions of the scheme are scalability, the requirement of only local knowledge of the desired formation topology and ease of reconfiguration in the face of loss of agents and/or channels.
\end{abstract}

1. Introduction. The cooperative control of mobile agents has become an important area of research in the past few years, ([1]-[30]). Aspects of the problem include: control with little or no centralized intervention, poor information quality, and performance of cooperative tasks.

This paper presents a framework for cooperative control of a fleet of agents with the objective of achieving and maintaining a prescribed formation. The goal of this work is to define a control law and associated communication architecture that derive directly from the manner in which the formation is specified. The class of formation considered here includes, but is not limited to, formations defined by arbitrary geometries that move with a constant velocity.

There are several papers in the broad area of controlling autonomous agents. One involves string stability, e.g. [22] concerning one dimensional strings of countably infinite autonomous objects functioning in a leader follower framework. The objective is to maintain a given distance between successive objects. The results require that each agent know the state of the leader. Here such a leader follower assumption is not needed. The interesting papers [25] and [12] have the goal of using nearest neighbor control to force all agents to move in a given direction. Placing the agents in prescribed relative positions is not an objective of [25] and [12]. Reference [30] concerns manifolds to which all agents must converge. It does require every agent to communicate with all others. Papers such as [29] concern maintaining formations defined by interagent distances, where only one agent is responsible for maintaining each distance.

\footnotetext{
* Dedicated to Brian Anderson on the occasion of his 70th birthday. Supported in part by NSF grants ECS-0622017, CCF-072902 and CCF-0830747.

$\dagger$ Authors are with the Department of of Electrical Computer Engineering, University of Iowa, 4016 Seamens Center, Iowa City, IA 52242, USA. E-mails: \{roabel, dasgupta, kuhl\}@engineering.uiowa.edu
} 


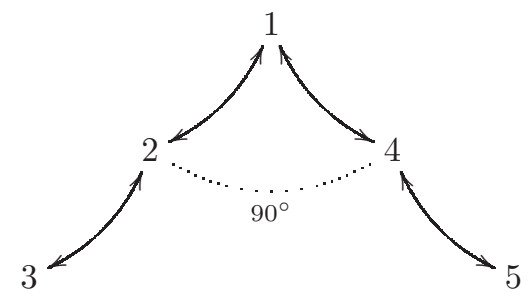

FIG. 1.1. agent Formation Topology with no Redundancy

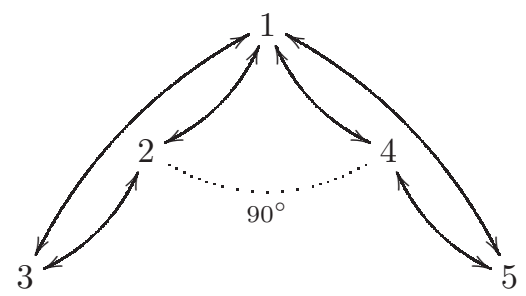

FIG. 1.2. agent Formation Topology with Redundancy

The papers closest to this work are [26], [7] and [8]. The emphasis in these papers has been to choose a communication architecture and a desired formation topology separately, and to decide whether or not the chosen communication architecture suffices to achieve the desired geometry. Thus in [8], the goal is to achieve formations with a prescribed set of relative positions. No velocity requirements are imposed, although in the noise free case all agents are asymptotically stationary. More importantly, the starting point is to specify a communication architecture and then provide sufficient (not necessary) conditions for this architecture to achieve stabilization.

In this paper we reverse the question and ask instead the following. Suppose one is given a required formation, what communication architecture is needed to achieve and maintain the specified topology? How much of the desired formation must be globally known? And how distributed a control law can be used?

The same geometry can be described in multiple ways. Thus if the desired geometry is that depicted in Figure 1.1 it can be described by specifying the relative positions between agents joined by arrows. Thus in this figure relative positions and/or relative velocities of the pairs $(1,2),(1,4),(2,3)$ and $(4,5)$ are specified. One may also specify the same geometry by adding redundant information, as in Figure 1.2, where the additional constraints are added between the pairs $(1,3)$ and $(1,5)$. Such a redundant structure adds fault tolerance to the geometric description. Thus, while the loss of agent 4 in Figure 1.1, implies that 5 is isolated, in Figure 1.2, 5 retains its position relative to agent 1 and the new topology remains viable. Thus additional fault tolerance is achieved in Figure 1.2 by adding redundancies in the geometric configuration such that the loss of any agent but 1 still results in an acceptable formation topology.

From here on we will call this the Formation Topology, as opposed to the Com- 
munication Topology which defines the state information flow required to implement a cooperative control law. We explore here the relation between these two topologies and argue that issues of fault tolerance, scalability and communication derive from the correct design of the formation topology.

To this end we propose a cost function that incorporates the formation topology. A one step ahead optimal control law obtained on its basis has many features. Foremost among them is the fact that the communication topology required to implement it is identical to the underlying formation topology.

A key technical result is that for the class of formation topologies here, an identical communication topology suffices to achieve it, iff the formation topology is viable. Viability means the existence of a centralized control law that asymptotically achieves and maintains the topology. For this class, distributed stabilization is possible as long as a centralized stabilizer can be enunciated. The resulting framework naturally incorporates robustness, scalability and fault-tolerance considerations and allows these to be addressed during the design of the formation topology itself.

The key attractive properties of our approach are as follows: In the sequel we will call a pair of agents "neighbors" if they appear in the same geometric constraint. Thus in Figure 1.1 Agent 1 has the neighbors 2, and 4, while in Figure 1.2 it has the additional neighbors 3 and 5 .

(a) Agent $i$ needs the state information of only its neighbors in the formation topology. Thus in Figure 1.1 agent 1 requires only the state information of 2 and 4, while in Figure 1.2 it also needs the state information of the two remaining agents. In both instances agent 2 requires only the state information of agents 1 and 3 .

(b) A given agent only needs to know the constraints imposed on itself by the formation topology. Thus in Figure 1.1, agent 2 needs only to know its desired position/velocity relative to 1 and 3 . Should the formation topology explicitly mandate that 2 move with a certain velocity, then of course 2 should be aware of this.

(c) Should the loss of an agent still permit a viable topology, e.g. the loss of 4 in Figure 1.2, then only the neighbors of the lost agent need to reconfigure their control law.

(d) Should the loss of a communication channel still permit a viable topology, e.g. the loss of the arc joining agents 1 and 5 in fig. 1.2, then only the agent at the end points of the lost arc need to reconfigure their control law.

(e) If a new agent joins the fleet by establishing a geometric position with respect to a subset of the agents, then only these agents need to reconfigure their control law.

(f) Relative position constraints can be augmented by compatible, potentially redundant velocity and/or relative velocity constraints. Thus one may impose 
a velocity requirement on agent 5 in Figure 1.2, that would automatically specify the direction and movement of the whole formation.

Thus (a) indicates the communication topology highlighted in the foregoing. Item (b) has the added attraction of permitting the control to be implemented by a given agent with only a local knowledge of the formation topology. Scalability comes from (e) as a new agent 6 in Figure 1.1 with only 5 as a neighbor would require that only 5 readjust its control law. Reconfurability under the loss of an agent is greatly facilitated.

2. Problem Description. When considering the problem of an $N$-agent formation our focus here is on a two dimensional formation topology, even though the ideas trivially extend to three dimensional formations as well. We shall partition the global, $4 N$ state vector $x$ of the network as

$$
x=\left[x_{1}^{T}, x_{2}^{T}\right]^{T},
$$

where $x_{1}$ and $x_{2}$ contain the positions and velocities respectively. In particular, denoting $x_{l, j}$ as the $j$-th element of $x_{l}$, we will have

$x_{1,2 i-1}$ is the $\mathrm{x}$ position of agent $i$,

$x_{2,2 i-1}$ is the $\mathrm{x}$ velocity of agent $i$,

$x_{1,2 i}$ is the y position of agent $i$, and

$x_{2,2 i}$ is the y velocity of agent $i$

For convenience we will denote

$$
n=2 N \text {. }
$$

We shall further assume that each agent has been internally controlled to represent a double integrator with elements $u_{2 i-1}$ and $u_{2 i}$ of the control input vector $u$ representing normalized force variables acting on the $i$-th agent, in the $x$ and $y$ directions respectively. For notational simplicity we will assume that the sampling interval is 1-second. The ideas trivially extend to nonunity sampling intervals. We note that all issues of the motion, such as non-holonomic properties etc are taken out of play here, as they would only serve to distract from the main thrust of the paper.

Thus, to within a suitable force normalization the system of agents can be described by:

$$
x(k+1)=\Phi x(k)+\Gamma u(k)
$$

where

$$
\Phi=\left[\begin{array}{cc}
I_{n} & I_{n} \\
0 & I_{n}
\end{array}\right] \text {, and } \Gamma=\left[\begin{array}{c}
I_{n} \\
2 I_{n}
\end{array}\right] .
$$

To ease notation we will often denote

$$
\Phi x[k]=\theta(k) .
$$


Using this equation we then have:

$$
x(k+1)=\theta(k)+\Gamma u(k) .
$$

Observe the following fact that follows directly from (2.3).

FACT 2.1. The $j$-th element of $\theta, \theta_{j}(k)$ for $j \in\{2 i-1,2 i, n+2 i-1, n+2 i\}$ requires only the states associated with agent $i$.

2.1. The formation topology. The formation topology will be characterized by two $L_{i} \times n$ matrices $A_{i}$ and two $L_{i} \times 1$ vectors $b_{i}$, designating separate constraints on the positions and velocities of the agents via the equations

$$
A_{i} x_{i}=b_{i}, \quad i \in\{1,2\} .
$$

With

$$
A=\left[\begin{array}{cc}
A_{1} & 0 \\
0 & A_{2}
\end{array}\right] \text { and } b=\left[\begin{array}{c}
b_{1} \\
b_{2}
\end{array}\right]
$$

the topology can be represented by the following equation:

$$
A x=b,
$$

where $x$ the state vector. In all there are $L_{1}$ position constraints and $L_{2}$ velocity constraints. Define the total number of constraints to be

$$
L=L_{1}+L_{2} .
$$

Observe that the relative positions between two agents $i$ and $j$ can be completely specified, for suitable $c$ and $d$ by the pair of equations

$$
x_{1,2 i-1}-x_{1,2 j-1}=c \text { and } x_{1,2 i}-x_{1,2 j}=d .
$$

Thus an arbitrary formation such as in Figure 1.1 or in Figure 1.2 can be specified by (2.6) for $i=1$. Indeed in Figure 1.1, $L_{1}$ is 8 and in Figure 1.2, $L_{1}$ is 12, which each row of $A_{1}$ having all but two elements zero and the remaining two being \pm 1 . One can add compatible absolute and/or relative velocity constraints through $A_{2}$ and $b_{2}$. Thus should either formation be required to fly with a constant velocity, then one can select, e.g.

$$
A_{2}=\left[I_{2}, 0, \cdots, 0\right]
$$

This would specify a velocity in $x$ and $y$ directions on agent 1 , which together with the relative position constraints, completely defines a formation flying with a constant velocity. One can add a redundancy in (2.11) by specifying also the velocity of 3, (to guard against the loss of either agent) by choosing

$$
A_{2}=\left[\begin{array}{llllll}
I_{2} & 0 & 0 & 0 & \cdots & 0 \\
0 & 0 & I_{2} & 0 & \cdots & 0
\end{array}\right] .
$$


Consider the formation topologies shown in Figure 1.1 and Figure 1.2. The arcs connecting the agents represent a position and/or velocity dependence between the representative agents in the topology. Indeed the following fact connects the existence of an arc in the formation topology with a property of $A$.

FACT 2.2. Define $a_{j l}$ as the $j, l$-th element of $A$. Then an arc exists between agents $p$ and $q$ iff for at least one $i \in\{2 p-1,2 p, n+2 p-1, n+2 p\}$ and $j \in$ $\{2 q-1,2 q, n+2 q-1, n+2 q\}$

$$
a_{l i} a_{l j} \neq 0 \text { for some } l \in\left\{1, \cdots, L_{1}+L_{2}\right\} .
$$

In other words there is at least one row of $A$ that contains nonzero entries from columns corresponding to velocities and/or positions of both $p$ and $q$.

Recall that while Figures 1.1 and 1.2 describe the same geometry the latter represents a formation topology with redundancies. Observe if the formation topologies in Figure 1.1 and Figure 1.2 are respectively defined by the pairs $\left[A^{(1)}, b^{(1)}\right]$ and $\left[A^{(2)}, b^{(2)}\right]$, then $\left[A^{(1)}, b^{(1)}\right]$ is a submatrix of $\left[A^{(2)}, b^{(2)}\right]$. Moreover, should the loss of an agent result in a topology that remains acceptable, e.g. the loss of 4 in Figure 1.2, then this new topology characterized by $\left[A^{(3)}, b^{(3)}\right]$ obtained by removing the rows corresponding to the constraints featuring 4 and columns corresponding to the states of 4 , is itself a submatrix of $\left[A^{(2)}, b^{(2)}\right]$. The loss of a communication channel, e.g. that between 1 and 5 would involve the use of a new pair obtained by removing rows characterizing the constraint defining this lost arc. This feature forms a core property to be exploited in fault tolerant design. Scalability is likewise incorporated rather easily. Thus if a new agent 6 appears in Figure 1.2 with an arc between it and 5, then the new pair $\left[A^{(4)}, b^{(4)}\right]$ characterizing it has $\left[A^{(2)}, b^{(2)}\right]$ as a submatrix, and involves just the addition of rows and columns, and aumenting rows in $\left[A^{(2)}, b^{(2)}\right]$ that feature in $\left[A^{(4)}, b^{(4)}\right]$ by zero column entries. In other words with $\times$ denoting arbitrary submatrices, one has

$$
\left[A^{(4)}, b^{(4)}\right]=\left[\begin{array}{ccc}
A^{(2)} & 0 & b^{(2)} \\
\times & \times & \times
\end{array}\right] .
$$

Thus the loss of an agent/communication channel requires working with a submatrix of the original $[A, b]$, and the addition of an agent requires a supermatrix of $[A, b]$.

2.2. Viability. In this section we explore conditions on $[A, b]$ under $(2.7)$ that ensures the viability of the formation topology (2.8). Before providing a formal definition and analysis of viability we first discuss at an intuitive level what it takes for a topology such as this to be both achieved and maintained.

Clearly at the minimum $b_{i}$ must be in range space of $A_{i}$. Secondly since $x_{2}$ is the derivative of $x_{1}$ and with $x_{i}$ defining a target formation, $A_{1} x_{1}$ is a constant,

$$
A_{1} x_{2}=0
$$


Finally it would be intutuitively appealing if once the formation is attained, it is maintained without any external force. In view of (2.2) this would require that for all nonnegative integers $m$

$$
A \Phi^{m} x=b .
$$

In a more formal sense we define a viable topolgy to be one that can be achieved and maintained by control law that may be centralized and even nonlinear time varying. More precisely:

Definition 2.1. Under (2.2) and (2.7), the formation topology (2.8) is viable if for every $x(0)$ there exists a bounded input sequence $u(k)$ such that.

$$
\lim _{k \rightarrow \infty}(A x(k)-b)=0 .
$$

The following theorem demonstrates that the intuitive properties we discussed at the outset of this subsection are necessary for viability.

TheOREm 2.1. Under (2.7) suppose (2.8) is viable. Then there exists $x$ as in (2.1) such that (2.16) holds for all nonnegative integers $m$ as does (2.18) below.

$$
A_{1} x_{2}=0
$$

Proof. If the topology is viable then (2.17) should hold. This implies that there exists $x$ as in (2.1) and an $[A, b]$ as in (2.7) such that

$$
A_{1} x_{1}=b_{1}
$$

and

$$
A_{2} x_{2}=b_{2}
$$

Also, using the same $x$ as above, under (2.2) and (2.17) there exists an input $u$ such that

$$
A[\Phi x+\Gamma u]=b .
$$

From (2.3) we have:

$$
\left[\begin{array}{cc}
A_{1} & 0 \\
0 & A_{2}
\end{array}\right]\left[\begin{array}{c}
x_{1}+x_{2}+u \\
x_{2}+2 u
\end{array}\right]=\left[\begin{array}{l}
b_{1} \\
b_{2}
\end{array}\right]
$$

It follows that:

$$
A_{1}\left(x_{2}+u\right)=0
$$

and

$$
A_{2} u=0 \text {. }
$$


Now if we define $\hat{x}$ using the same $x$ and $u$ as above

$$
\hat{x}=\left[\begin{array}{c}
x_{1} \\
x_{2}+u
\end{array}\right]
$$

we have

$$
A \hat{x}=\left[\begin{array}{cc}
A_{1} & 0 \\
0 & A_{2}
\end{array}\right]\left[\begin{array}{c}
x_{1} \\
x_{2}+u
\end{array}\right]=\left[\begin{array}{l}
b_{1} \\
b_{2}
\end{array}\right]=b
$$

since $A_{2} u=0$ from (2.24). Also because (2.23) we have

$$
A \Phi^{m} \hat{x}=b \quad \forall m \in \mathbb{N} \cup\{0\} .
$$

This can easily be verified since

$$
\Phi^{m}=\left[\begin{array}{cc}
I_{n} & m I_{n} \\
0 & I_{n}
\end{array}\right] .
$$

Therefore (2.27) can be rewritten as

$$
\left[\begin{array}{cc}
A_{1} & 0 \\
0 & A_{2}
\end{array}\right]\left[\begin{array}{c}
x_{1}+m\left(x_{2}+u\right) \\
x_{2}+u
\end{array}\right]=b \quad \forall m \in \mathbb{N} \cup\{0\}
$$

Finally, since there exists an $\hat{x}$ such that (2.27) holds the input force becomes 0 , and $A_{1} x_{2}=0$ in $(2.23)$.

Thus the existence of a control law necessitates the intuitive conditions we stated earlier. Indeed we go on to show that these conditions suffice for the attainment and maintenance of the formation topology and enable these tasks through a communication topology that mirrors the formation topology defining the formation.

3. Control Law and Communication Topology. We propose a one step ahead optimization law using the cost function

$$
\begin{array}{r}
J(k)=[A x(k+1)-b]^{T}[A x(k+1)-b]+ \\
+u^{T}(k) Q u(k)
\end{array}
$$

Where $Q=Q^{T}>0$ penalizes the input. The key step in achieving the control law with the desired characteristics described in the introduction is to appropriately select Q.

Since $x(k+1)$ is dependent on $u(k)$ we begin by substituting $(2.2,2.4)$ into the cost function defined in (3.1). Taking the partial derivative of the resultant expression with respect to $u(k)$, we obtain:

$$
\left[\Gamma^{T} A^{T} A \Gamma+Q\right] u(k)=\Gamma^{T} A^{T}[b-A \theta(k)]
$$


Setting:

$$
Q=\alpha I-\Gamma^{T} A^{T} A \Gamma
$$

with $\alpha$ greater than the largest eigenvalue of $\Gamma^{T} A^{T} A \Gamma, Q$ is invertible and positive definite. Further by making $\alpha$ arbitrarily large one can penalize the input to an arbitrary degree. The resulting control law is shown below.

$$
u(k)=\frac{1}{\alpha} \Gamma^{T} A^{T} b-\frac{1}{\alpha} \Gamma^{T} A^{T} A \theta(k)
$$

Now we will show that the communication topology resulting from (3.4) is identical to the geometric topology and further that only a local knowledge of the formation is required by each agent. Observe that the control inputs to agent $i$ are $u_{2 i}$ and $u_{2 i-1}$. We will show that if $i$ and $j$ do not have an arc between them in the formation topology, then $u_{2 i}$ and $u_{2 i-1}$ do not depend on $\theta_{2 j-1}, \theta_{2 j}, \theta_{2 j-1+n}$ and $\theta_{n+2 j}$. Because of Fact 2.1 this in turn implies that $u_{2 i}$ and $u_{2 i-1}$ do not depend on $x_{2 j-1}, x_{2 j}, x_{2 j-1+n}$ and $x_{n+2 j}$, establishing the structure of the communication topology. Observe that (3.4) becomes,

$$
u(k)=\frac{A_{1}^{T} b_{1}+2 A_{2}^{T} b_{2}-\left[A_{1}^{T} A_{1}, 2 A_{2}^{T} A_{2}\right] \theta(k)}{\alpha}
$$

We next present the following Lemma.

Lemma 3.1. For any matrix $C$

$$
\left(C^{T} C\right)_{i j} \neq 0
$$

only if for some l

$$
c_{l i} c_{l j} \neq 0
$$

Further, the computation of the $i$-th row of $C^{T} C$ requires the knowledge of the l-th row of $C$ only if $c_{l i} \neq 0$. Finally, for any vector $g$ the computation of the $i$-th element of $C^{T} g$ requires the knowledge of the $l$-th row of $C$ and/or l-th element of $g$, only if $c_{l i} \neq 0$.

Proof. Follows from the fact that

$$
\left(C^{T} C\right)_{i j}=\sum_{l} c_{l i} c_{l j}
$$

and

$$
\left(C^{T} g\right)_{i}=\sum_{l} c_{l i} g_{l}
$$

Then we have the following result that establishes the various properties of the communication topology listed in the foregoing.

ThEOREM 3.2. Consider (3.4) under (2.1), (2.3), and (2.7). Then the finding $u_{2 i-1}(k)$ and $u_{2 i}(k)$ requires: 
(A) The states of agent $l$ only if there is an arc between agents $l$ and $i$ in the formation topology.

(B) The $l$-th row of $A$ only if for some $j \in\{2 i-1,2 i, 2 i-1+n, 2 i+n\} a_{l j} \neq 0$.

(C) The l-th element of $b$ only if for some $j \in\{2 i-1,2 i, 2 i-1+n, 2 i+n\} a_{l j} \neq 0$.

Proof. Consider the determination of $u_{p}, p \in\{2 i-1,2 i\}$. Suppose this requires the knowledge of $\theta_{q}$, for some $q \in\{2 l-1,2 l\}$. Then from (3.5) $\left(A_{1}^{T} A_{1}\right)_{p q}$ is non zero. Then because of Lemma 3.1 for at least one $m$ the $m$-th row of $A_{1}$ must have nonzero entries in both the $p$-th and the $q$-th locations. Similarly if the determination of $u_{p}$, $p \in\{2 i-1,2 i\}$ requires the knowledge of $\theta_{q}$, for some $q \in\{2 l-1+n, 2 l+n\}$, then for at least one $m$ the $m$-th row of $A_{2}$ has nonzero entries in both the $p$-th and the $q$-th locations. Then facts 2.1 and 2.2, together with (2.7) prove (A).

Now, from (3.5), the computation of $u_{p}, p \in\{2 i-1,2 i\}$ requires the computation of the $p$-th rows of $A_{1}^{T} A_{1}$ and $A_{2}^{T} A_{2}$ and the $p$-th elements of $A_{1}^{T} b_{1}$ and $A_{2}^{T} b_{2}$. Then from Lemma 3.1, for such a $p$, the computation of the $p$-th rows of $A_{1}^{T} A_{1}$ and $A_{2}^{T} A_{2}$ require respectively, the knowledge of the $l$-th rows of $A_{1}$ and $A_{2}$ only if the $l p$-th elements of respectively $A_{1}$ and $A_{2}$ are nonzero. Further the $p$-th elements of $A_{1}^{T} b_{1}$ and $A_{2}^{T} b_{2}$ require the knowledge of $l$-th rows of $A_{1}$ and $A_{2}$ and/or the $l$-th elements of $b_{1}$ and $b_{2}$ only if the $l p$-th elements of respectively $A_{1}$ and $A_{2}$ are nonzero. Then (B) and (C) follow from (2.7), fact 2.1 and (2.1).

(A) shows that the communication topology is the same as the formation topology. (B) and (C) show that agent $i$ need only know those rows of $A$ and elements of $b$ which define the arcs emanating from it. Thus $i$ must only know its place in the formation topology and therefore, a distributed knowledge of the formation topology suffices.

If despite the loss of an agent, e.g. 4 in Figure 1.2, the formation topology remains viable, then this modified formation topology is described by a $[A, b]$ matrix that is a submatrix of its counterpart in the original formation topology, and obtained by removing the rows characterizing the two arcs impacting 4 and the four columns of $A$ corresponding to the states of 4 . As the elements of these columns in the rows of the original $A$ matrix defining the arcs of 2 and 3 are zero, the inputs to agents 2 and 3 are unchanged. These agents do not reconfigure their control laws and need not know about the loss. Similarly if communication between 1 and 5 be impaired or lost, then only 1 and 5 must know of this loss and adjust their control law.

Scalability is similarly accomodated. The position of new arrival can be completely specified by introducing an arc to a single member of the formation. Then because of the relation between the larger $[A, b]$ matrix describing the augmented formation topology, and the old one, see e.g. (2.14), none of the elements of this new $[A, b]$ matrix affect the control laws of the remaining agents.

Evidently, these attractive properties stem from the choice of an one step ahead optimal control law and the judicious selection of $Q$ the matrix that penalizes large control action. The fact that one step ahead laws with the right cost function lead 
to attractive features has been noted before in the adaptive control literature, [3] and [13], where convergence depended on these choices.

4. Proof of Stability. In this section we prove that the control law in (3.4) asymptotically attains all viable formation topologies, as long as

$$
I-\frac{\Gamma^{T} A^{T} A \Gamma}{\alpha}>0 .
$$

To this end observe that with

$$
F=\Phi-\frac{\Gamma}{\alpha} \Gamma^{T} A^{T} A \Phi,
$$

and

$$
G=\frac{\Gamma}{\alpha} \Gamma^{T} A^{T} b,
$$

the control law (3.4) results in the closed loop

$$
x(k+1)=F x(k)+G .
$$

Define

$$
y(k)=A x(k)-b .
$$

We need to find conditions under which $y(k)$ asymptotically approaches zero. To this end we first provide the following lemma.

Lemma 4.1. Under (4.1), with $F$ defined in (4.2), (2.3) and $A$ in (2.7), all poles of $A(z I-F)^{-1}$ are inside the unit circle.

Proof. The detailed proof is in the appendix and comprises two parts. In the first we show that (4.1) ensures that the poles of $F$ are either at 1 or inside the unit circle. The second part shows that the poles at 1 are unobservable through $A$.

This brings us to the main result of this section.

Theorem 4.2. Suppose the formation topology is viable and $A_{1} \neq 0$. Then

$$
\lim _{k \rightarrow \infty} A x(k)=b
$$

Proof. We need to show that

$$
\begin{aligned}
R(z) & =\frac{z-1}{z}\left[A(z I-F)^{-1} x(0)+\right. \\
& \left.+A(z I-F)^{-1} \frac{G z}{z-1}-\frac{b z}{z-1}\right]
\end{aligned}
$$

is analytic on or inside the unit circle and

$$
\lim _{z \rightarrow 1} R(1)=0 .
$$

Because of Lemma $4.1 A(z I-F)^{-1}$ and hence $R(z)$ is analytic on or outside the unit circle. Thus it suffices to show that

$$
\lim _{z \rightarrow 1}\left[A(z I-F)^{-1} G-b\right]=0
$$


Since the formation topology is viable there exists an $x$ as in (2.1) that satisfies the constraints imposed by Theorem 2.1. For such an $x$ and all nonnegative integer $l$, from Theorem 2.1, we have

$$
\begin{array}{r}
A \Phi^{l}\left[\begin{array}{c}
x_{2} \\
0
\end{array}\right]=\left[\begin{array}{cc}
A_{1} & 0 \\
0 & A_{2}
\end{array}\right]\left[\begin{array}{cc}
I & l I \\
0 & I
\end{array}\right]\left[\begin{array}{c}
x_{2} \\
0
\end{array}\right]= \\
=A_{1} x_{2}=0 .
\end{array}
$$

Further for such an $x$ and all nonegative integer $m$, we have from (4.2) and (4.7) that

$$
\begin{array}{r}
A F^{m}\left[\begin{array}{c}
x_{2} \\
0
\end{array}\right]=A F^{m-1}\left[I-\frac{\Gamma \Gamma^{T} A^{T} A}{\alpha}\right] \Phi\left[\begin{array}{c}
x_{2} \\
0
\end{array}\right] \\
=A F^{m-1} \Phi\left[\begin{array}{c}
x_{2} \\
0
\end{array}\right]
\end{array}
$$

Thus by induction and (4.7) for all nonnegative integer $m$

$$
A F^{m}\left[\begin{array}{c}
x_{2} \\
0
\end{array}\right]=A \Phi^{m}\left[\begin{array}{c}
x_{2} \\
0
\end{array}\right]=0 .
$$

Since $A \Phi^{m} x=b$ for all nonnegative integer $m$,

$$
\begin{aligned}
& \lim _{z \rightarrow 1} A(z I-F)^{-1} G-b \\
& =\lim _{z \rightarrow 1} A(z I-F)^{-1} G-A x \\
& =\lim _{z \rightarrow 1}\left[A(z I-F)^{-1}\{G-(z I-F) x\}\right] \\
& =\lim _{z \rightarrow 1}\left[A(z I-F)^{-1} \times\right. \\
& \left.\quad \times\left\{\frac{\Gamma \Gamma^{T} A^{T} A}{\alpha} x-x+\Phi x-\frac{\Gamma \Gamma^{T} A^{T} A \Phi x}{\alpha}\right\}\right] \\
& =\lim _{z \rightarrow 1}\left[A(z I-F)^{-1}\{-x+\Phi x\}\right] \\
& =\lim _{z \rightarrow 1}\left[A(z I-F)^{-1}\left[\begin{array}{c}
x_{2} \\
0
\end{array}\right]\right] .
\end{aligned}
$$

We will now show that in fact

$$
A(z I-F)^{-1}\left[\begin{array}{c}
x_{2} \\
0
\end{array}\right]=0
$$


almost every where. Thus as it is rational it is zero every where including at $z=1$. Indeed in the region of convergence of $(z I-F)^{-1}$.

$$
\begin{array}{r}
A(z I-F)^{-1}\left[\begin{array}{c}
x_{2} \\
0
\end{array}\right]=A z^{-1}\left(I-z^{-1} F\right)^{-1}\left[\begin{array}{c}
x_{2} \\
0
\end{array}\right] \\
=\left[A z^{-1}+z^{-1} \sum_{i=1}^{\infty} z^{-i} A F^{i}\right]\left[\begin{array}{c}
x_{2} \\
0
\end{array}\right] \\
=z^{-1} \sum_{i=1}^{\infty} z^{-i} A F^{i}\left[\begin{array}{c}
x_{2} \\
0
\end{array}\right] \\
=0
\end{array}
$$

where the last equality follows from (4.8).

Thus this distributed control law helps attain and maintain all viable formation topologies. Three implications of this result bear reiteration. First, the necessary conditions for viablity given in Theorem 2.1 are all that are invoked in the proof of Theorem 4.2. Thus these necessary conditions are also sufficient for viability. Second, it is easily seen from the proof of Theorem 4.2 that in fact

$$
\lim _{k \rightarrow \infty} u(k)=0 .
$$

In other words once the formation is attained it can be maintained with no control input. Finally, and more compellingly, the class of formation topology under consideration here has the attractive property that a distributed control law for its achievement exists, as long as a centralized law exists. Thus whatever can be done through global action can also be achieved through local action, and as importantly through local knowledge of the overall objective.

5. Simulations. We assume that there are no velocity constraints i.e. $A_{2}=0$. Relative positions are specified using equations of the form in (2.10).

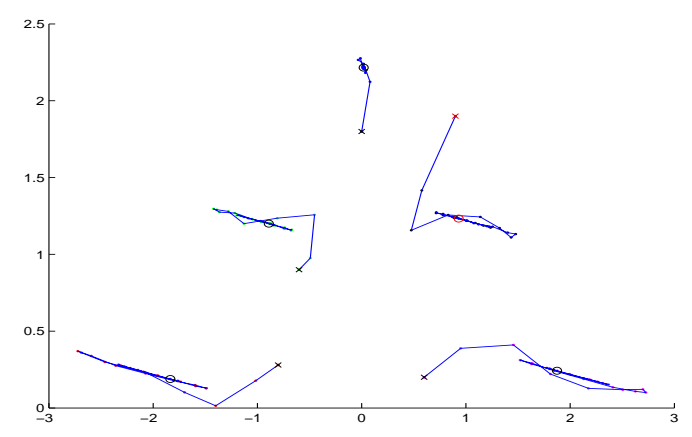

FIG. 5.1. Agent formation with no redundancy 
In all the simulations, the initial conditions of the fleet are the same. The starting positions are denoted by an $\times$, the positions at each time step are denoted by a $\cdot$, and the final positions are denoted by a $\circ$. All simulations are run until the desired formation is reached.

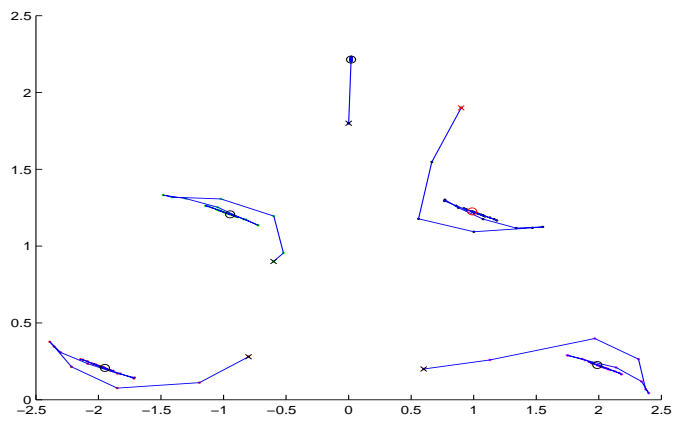

FIG. 5.2. Agent formation with redundancy

Figure 5.1 corresponds to Figure 1.1. Figure 5.2 shows the motion of the fleet with the additional redundancy defined in Figure 1.2, which provides additional relative state information to agents 3 and 5 . By inspection, one can notice less oscillation in Figure 5.2 as compared to Figure 5.1. Figure 5.4 shows the position errors $\|A x-b\|$ of the fleet for Figures 5.1 and 5.2. The dashed line with o represents the redundant formation, and the solid line with squares represents the non-redundant formation. Notice that a redundant formation topology has the added advantage of faster convergence, even without the loss of agent, i.e. it is more robust from a performance point of view as well.

The effects of a lost agent for the setting of Figure 5.2 can be seen in Figure 5.3. In this example agent 4 is lost after 4 time steps. The position at which agent 4 is lost is denoted by a $*$. Once a loss has been detected, the rows corresponding to the constraints of the lost agent, and the columns associated with its states are removed.

6. Conclusions. We have examined the optimal cooperative control of a fleet of automomous units and proposed an optimal control strategy that results in distributed control, requiring a communication topology that mirrors exactly the formation topology. We have concluded that fault tolerant design must be incorporated in the formation topology alone, and the rest will follow from the control law itself. Our control law permits easy reconfiguration in response to lost agent, is scalable, and requires only the local knowledge of the formation topology. The key to achieving these properties was in the judicious selection of the cost function to be optimized. Though we have not analyzed the effect of transmission delays, we conjecture that as 


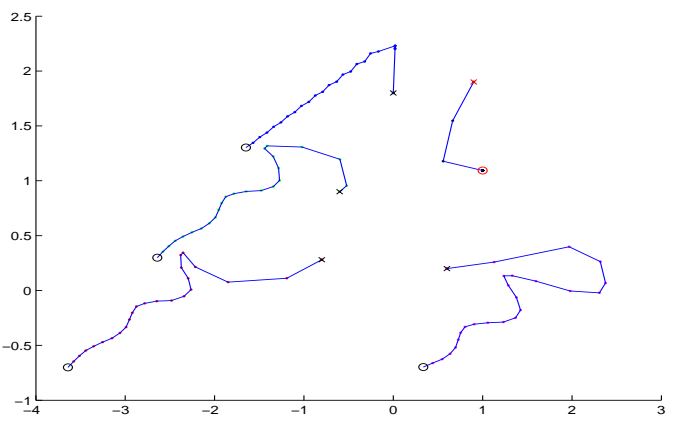

FIG. 5.3. Agent formation with redundancy and the loss of agent 4 at time $k=5$

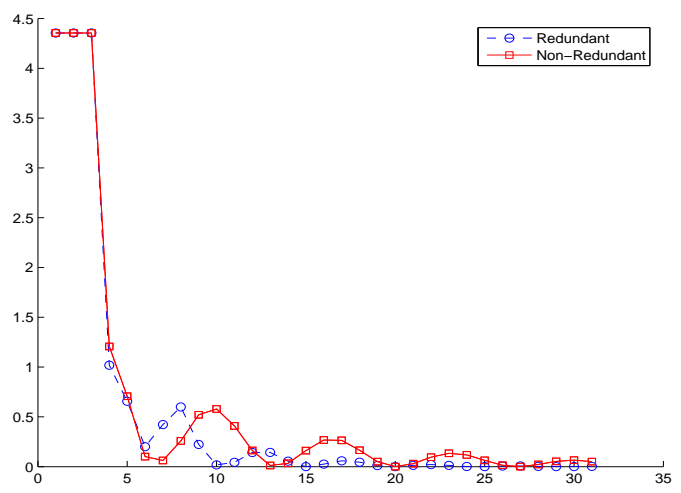

FIG. 5.4. Difference in the formation error with a redundant topology and a non-redundant topology

with similar algorithms, e.g. [19], there will be some robustness to modest delays.

\section{Appendix A. Proof of Lemma 4.1.}

We first complete through the lemma below the first part of the proof of Lemma 4.1 .

Lemma A.1. Under (2.7) (4.1)-(4.3), and (2.3), the poles of $A(z I-F)^{-1}$ are either inside the unit circle or at 1.

Proof. Choose:

$$
T=\left[\begin{array}{cc}
I & -I / 2 \\
0 & I
\end{array}\right] \text { and hence } T^{-1}=\left[\begin{array}{cc}
I & I / 2 \\
0 & I
\end{array}\right] .
$$

Call

$$
\Sigma_{i}=A_{i}^{T} A_{i} \geq 0
$$


Because of (2.3) and (4.1)

$$
0 \leq \frac{\Sigma_{1}+4 \Sigma_{2}}{\alpha}<1
$$

Then

$$
\begin{aligned}
\text { TFT }^{-1} & =\Phi-\frac{1}{\alpha}\left[\begin{array}{c}
0 \\
2 I
\end{array}\right]\left[\begin{array}{cc}
\Sigma_{1} & 2 \Sigma_{2}
\end{array}\right]\left[\begin{array}{cc}
I & \frac{3}{2} I \\
0 & I
\end{array}\right] \\
& =\Phi-\frac{1}{\alpha}\left[\begin{array}{c}
0 \\
2 I
\end{array}\right]\left[\begin{array}{cc}
\Sigma_{1} & \frac{3}{2} \Sigma_{1}+2 \Sigma_{2}
\end{array}\right] \\
& =\left[\begin{array}{cc}
I & I \\
-\frac{2 \Sigma_{1}}{\alpha} & I-\frac{3 \Sigma_{1}+4 \Sigma_{2}}{\alpha}
\end{array}\right] .
\end{aligned}
$$

Then

$$
\begin{aligned}
& \operatorname{det}\left(z I-T F T^{-1}\right) \\
& =\operatorname{det}\left[(z-1)^{2} I+(z-1) \frac{3 \Sigma_{1}+4 \Sigma_{2}}{\alpha}+\frac{2 \Sigma_{1}}{\alpha}\right] \\
& =\operatorname{det}\left[z^{2} I+\left(\frac{3 \Sigma_{1}+4 \Sigma_{2}}{\alpha}-2 I\right) z-\left(\frac{\Sigma_{1}+4 \Sigma_{2}}{\alpha}-I\right)\right]
\end{aligned}
$$

Then if $z$ is an eigenvalue of $F$, there exists a unit $\eta$ such that

$$
z^{2}+\left(3 \sigma_{1}^{2}+4 \sigma_{2}^{2}-2\right) z-\left(\sigma_{1}^{2}+4 \sigma_{2}^{2}-1\right)=0
$$

where

$$
\sigma_{i}^{2}=\frac{\eta^{T} \sum_{i} \eta}{\alpha}
$$

From (A.1)

$$
0 \leq \sigma_{1}^{2}+4 \sigma_{2}^{2}<1
$$

and therefore

$$
0 \leq 3 \sigma_{1}^{2}+4 \sigma_{2}^{2}<1+2 \sigma_{1}^{2}
$$

First observe that if (A.2) has a complex root $\rho$ then from (A.3)

$$
|\rho|^{2}=1-\sigma_{1}^{2}-4 \sigma_{2}^{2} \leq 1
$$

with equality holding iff $\sigma_{1}=\sigma_{2}=0$, which in turn leads to

$$
\rho=1 .
$$

If on the other hand the roots are real then they are

$$
\rho_{1}=\frac{2-3 \sigma_{1}^{2}-4 \sigma_{2}^{2}+\sqrt{\left(3 \sigma_{1}^{2}+4 \sigma_{2}^{2}\right)^{2}-8 \sigma_{1}^{2}}}{2}
$$


and

$$
\rho_{2}=\frac{2-3 \sigma_{1}^{2}-4 \sigma_{2}^{2}-\sqrt{\left(3 \sigma_{1}^{2}+4 \sigma_{2}^{2}\right)^{2}-8 \sigma_{1}^{2}}}{2}
$$

Clearly $\rho_{1} \leq 1$, and because of (A.4) $\rho_{1}>-\frac{1}{2}$ as shown below

$$
\rho_{1} \geq \frac{2-\left(3 \sigma_{1}^{2}+4 \sigma_{2}^{2}\right)}{2}>\frac{1}{2}-\sigma_{1}^{2} \geq-\frac{1}{2}
$$

Further $\rho_{2} \leq 1$. Now assume

$$
\begin{aligned}
\rho_{2} & \leq-1 \\
\Leftrightarrow 4-\left(3 \sigma_{1}^{2}+4 \sigma_{2}^{2}\right) & \leq \sqrt{\left(3 \sigma_{1}^{2}+4 \sigma_{2}^{2}\right)^{2}-8 \sigma_{1}^{2}} \\
\Leftrightarrow 16-8\left(3 \sigma_{1}^{2}+4 \sigma_{2}^{2}\right) & \leq-8 \sigma_{1}^{2} \\
\Leftrightarrow 1 & \leq \sigma_{1}^{2}+2 \sigma_{2}^{2}
\end{aligned}
$$

But by (A.4), $1>\sigma_{1}^{2}+4 \sigma_{2}^{2}$, leading to a contradiction.

We next need to show that the eigenvalues of $F$ that are at 1 , are in fact unobservable from $A$. To this end we consider a singular value decomposition (SVD) of $A_{1}$, i.e. with $U_{1}, L_{1} \times L_{1}, V_{1}, n \times n$ unitary matrices

$$
A_{1}=U_{1} D_{1} V_{1}
$$

where

$$
D_{1}=\left[\begin{array}{cc}
\Lambda_{1} & 0 \\
0 & 0
\end{array}\right]
$$

and $\Lambda_{1}$ is diagonal, $n_{1} \times n_{1}$, positive definite.

Partition the $L_{2} \times n$ matrix $A_{2} V_{1}^{H}$ as

$$
A_{2} V_{1}^{H}=\left[\begin{array}{ll}
\bar{A}_{21} & \bar{A}_{22}
\end{array}\right]
$$

where $\bar{A}_{22}$ is $L_{2} \times\left(n-n_{1}\right)$.

Consider next the SVD of $\bar{A}_{22}$ i.e. with $U_{2}, L_{2} \times L_{2}$ and $V_{2},\left(n-n_{1}\right) \times\left(n-n_{1}\right)$ and both unitary

$$
\bar{A}_{22}=U_{2} D_{2} V_{2}
$$

where

$$
D_{2}=\left[\begin{array}{cc}
\Lambda_{2} & 0 \\
0 & 0
\end{array}\right]
$$

with $\Lambda_{2}, n_{2} \times n_{2}$, positive definite.

Define

$$
W=V_{1}^{H}\left[\begin{array}{cc}
I_{n_{1}} & 0 \\
0 & V_{2}^{H}
\end{array}\right]
$$


In the sequel $\oplus$ will denote the direct sum e.g. $A=A_{1} \oplus A_{2}$. Consider next a system equivalent to (4.4).

Lemma A.2. With $\Phi, \Gamma, A, b, F, G, U_{i}, V_{i} D_{i}, \bar{A}_{22}$ defined in (2.3), (2.7), (4.2), (4.3) and (A.9-A.14) define

$$
\begin{gathered}
\widehat{F}=\Phi-\frac{\Gamma \Gamma^{T} \widehat{A}^{T} \widehat{A} \Phi}{\alpha} \\
\widehat{A}=\left(U_{1} D_{1}\right) \oplus\left[\bar{A}_{21}, U_{2} D_{2}\right] \\
\widehat{G}=\frac{\Gamma \Gamma^{T} \widehat{A}^{T} b}{\alpha} \\
\widehat{x}(k)=\left(W^{H} \oplus W^{H}\right) x(k) .
\end{gathered}
$$

Then one has that

$$
\begin{gathered}
\widehat{x}(k+1)=\widehat{F} \widehat{x}(k)+\widehat{G} \\
y(k)=\widehat{A} \widehat{x}(k)-b
\end{gathered}
$$

Proof. First note that

$$
\begin{aligned}
A(W \oplus W) & =\left[\begin{array}{cc}
A_{1} V_{1}^{H} & 0 \\
0 & A_{2} V_{1}^{H}
\end{array}\right]\left[\begin{array}{cccc}
I & 0 & 0 & 0 \\
0 & V_{2}^{H} & 0 & 0 \\
0 & 0 & I & 0 \\
0 & 0 & 0 & V_{2}^{H}
\end{array}\right] \\
& =\left[U_{1}\left[\begin{array}{cc}
\Lambda_{1} & 0 \\
0 & 0
\end{array}\right]\right] \oplus\left[\bar{A}_{21}, U_{2} D_{2}\right] \\
& =\widehat{A} .
\end{aligned}
$$

Further

$$
\begin{aligned}
& \left(W^{H} \oplus W^{H}\right) F(W \oplus W)= \\
& =\left(\begin{array}{cc}
W^{H} & 0 \\
0 & W^{H}
\end{array}\right)\left(I-\frac{1}{\alpha} \Gamma \Gamma^{T} A^{T} A\right)\left[\begin{array}{cc}
I & I \\
0 & I
\end{array}\right]\left[\begin{array}{cc}
W & 0 \\
0 & W
\end{array}\right] \\
& =\left(\begin{array}{cc}
W^{H} & 0 \\
0 & W^{H}
\end{array}\right)\left(I-\frac{1}{\alpha}\left[\begin{array}{cc}
I & 2 I \\
2 I & 4 I
\end{array}\right] A^{T} A\right)\left[\begin{array}{cc}
W & 0 \\
0 & W
\end{array}\right] \Phi \\
& =\left(I-\frac{1}{\alpha} \Gamma \Gamma^{T}\left(\begin{array}{cc}
W^{H} & 0 \\
0 & W^{H}
\end{array}\right) A^{T} A\left[\begin{array}{cc}
W & 0 \\
0 & W
\end{array}\right]\right) \Phi \\
& =\widehat{F}
\end{aligned}
$$


because of (2.7). Similarly, because of (2.7), (2.3) and (4.3)

$$
\widehat{G}=\left(W^{H} \oplus W^{H}\right) G .
$$

Thus the Lemma holds.

We next show that a condition comparable to (4.1) holds.

Lemma A.3.

$$
I-\frac{\Gamma^{T} \widehat{A}^{T} \widehat{A} \Gamma}{\alpha}>0
$$

Proof. Follows from (A.21), the fact that

$$
W^{H} W=I
$$

and that

$$
\Gamma W=(W \oplus W) \Gamma
$$

Denoting $0_{p}$ to be the $p \times p, 0$ matrix, we observe from (A.10), (A.13) and (A.16):

$$
\begin{aligned}
\widehat{A}^{T} \widehat{A}= & {\left[\begin{array}{cc}
D_{1}^{H} U_{1}^{H} & 0 \\
0 & \bar{A}_{21}^{T} \\
0 & D_{2}^{H} U_{2}^{H}
\end{array}\right]\left[\begin{array}{ccc}
U_{1} D_{1} & 0 & 0 \\
0 & \bar{A}_{21} & U_{2} D_{2}
\end{array}\right] } \\
= & {\left[\begin{array}{ccccc}
\Lambda_{1}^{2} & 0 & 0 & 0 & 0 \\
0 & 0_{n-n_{1}} & 0 & 0 & 0 \\
0 & 0 & \bar{A}_{21}^{T} \bar{A}_{21} & B & 0 \\
0 & 0 & B^{H} & \Lambda_{2}^{2} & 0 \\
0 & 0 & 0 & 0 & 0_{n-n_{1}-n_{2}}
\end{array}\right], }
\end{aligned}
$$

where

$$
B=\bar{A}_{21}^{T} U_{2}\left[\begin{array}{c}
\Lambda_{2} \\
0
\end{array}\right] .
$$

Further,

$$
\begin{gathered}
\Gamma^{T} \widehat{A}^{T} \widehat{A}= \\
{\left[\begin{array}{cccccc}
\Lambda_{1}^{2} & 0 & 0 & 2 \bar{A}_{21}^{T} \bar{A}_{21} & 2 B & 0 \\
0 & 0_{n_{2}} & 0 & 2 B^{H} & 2 \Lambda_{2}^{2} & 0 \\
0 & 0 & 0_{n-n_{1}-n_{2}} & 0 & 0 & 0_{n-n_{1}-n_{2}}
\end{array}\right]}
\end{gathered}
$$


and

$$
\begin{aligned}
& \Gamma^{T} \widehat{A}^{T} \widehat{A} \Phi= \\
& {\left[\begin{array}{cccccc}
\Lambda_{1}^{2} & 0 & 0 & \Lambda_{1}^{2}+2 \bar{A}_{21}^{T} \bar{A}_{21} & 2 B & 0 \\
0 & 0 & 0 & 2 B^{H} & 2 \Lambda_{2}^{2} & 0 \\
0 & 0 & 0 & 0 & 0 & 0_{n-n_{1}-n_{2}}
\end{array}\right]}
\end{aligned}
$$

Then

$$
\widehat{F}=\left[\begin{array}{cc}
\widehat{F}_{11} & \widehat{F}_{12} \\
\widehat{F}_{21} & \widehat{F}_{22}
\end{array}\right]
$$

Where:

$$
\begin{aligned}
& \widehat{F}_{11}=\left[\begin{array}{ccc}
I_{n_{1}}-\frac{\Lambda_{1}^{2}}{\alpha} & 0 & 0 \\
0 & I_{n_{2}} & 0 \\
0 & 0 & I_{n-n_{1}-n_{2}}
\end{array}\right] \\
& \widehat{F}_{12}=\left[\begin{array}{ccc}
I_{n_{1}}-\frac{\Lambda_{1}^{2}+2 \bar{A}_{21}^{T} \bar{A}_{21}}{{ }^{\alpha}} & -\frac{2 B}{\alpha} & 0 \\
-\frac{2 B^{H}}{\alpha} & I_{n_{2}}-\frac{2 \Lambda_{2}^{2}}{\alpha} & 0 \\
0 & & I_{n-n_{1}-n_{2}}
\end{array}\right]
\end{aligned}
$$

$$
\begin{aligned}
& \widehat{F}_{21}=\left[\begin{array}{ccc}
-\frac{2 \Lambda_{2}^{2}}{\alpha} & 0 & 0 \\
0 & 0 & 0 \\
0 & 0 & 0
\end{array}\right] \\
& \widehat{F}_{22}=\left[\begin{array}{ccc}
I_{n_{1}}-\frac{\Lambda_{1}^{2}+4 \bar{A}_{21}^{T} \bar{A}_{21}}{\frac{4 B^{H}}{\alpha}} & -\frac{4 B}{\alpha} & 0 \\
-\frac{4 \Lambda_{2}^{2}}{\alpha} & 0 \\
0 & & I_{n-n_{1}-n_{2}}
\end{array}\right]
\end{aligned}
$$

Notice (A.23) and (A) imply

$$
\frac{4 \Lambda_{2}^{2}}{\alpha}<I
$$

Then the following lemma goes toward a Kalman like decomposition.

Lemma A.4. Under (A.9-A.16) with $L$ as in (2.9)

$$
\widehat{A}(z I-\widehat{F})^{-1}=\left[\begin{array}{ll}
H(z) & 0_{L \times\left(2 n-2 n_{1}-n_{2}\right)}
\end{array}\right] \Pi
$$

where

$$
\begin{gathered}
H(z)=C(z I-\Upsilon)^{-1} \\
\Upsilon=\left[\begin{array}{ccc}
I-\frac{\Lambda_{1}^{2}}{\alpha} & I-\frac{\Lambda_{1}^{2}+2 \bar{A}_{21}^{T} \bar{A}_{21}}{\alpha} & -\frac{2 B}{\alpha} \\
-\frac{2 \Lambda_{1}^{2}}{\alpha} & I-\frac{2 \Lambda_{1}^{2}+4 \bar{A}_{21}^{T} \bar{A}_{21}}{\alpha} & -\frac{4 B}{\alpha} \\
0 & -\frac{4 B^{H}}{\alpha} & I-\frac{4 \Lambda_{2}^{2}}{\alpha}
\end{array}\right],
\end{gathered}
$$




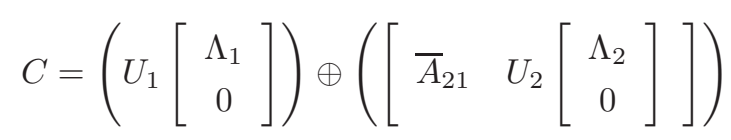

and

$$
\Pi=\left[\begin{array}{cccc}
I_{n_{1}} & 0 & 0 & 0 \\
0 & 0 & I_{n_{1}+n_{2}} & 0 \\
0 & I_{n-n_{1}} & 0 & 0 \\
0 & 0 & 0 & I_{n-\left(n_{1}+n_{2}\right)}
\end{array}\right]
$$

Proof. Note

$$
\Pi^{T} \Pi=I
$$

Hence

$$
\widehat{A}(z I-\widehat{F})^{-1}=\widehat{A} \Pi^{T}\left[z I-\Pi \widehat{F} \Pi^{T}\right]^{-1} \Pi .
$$

Now,

$$
\begin{aligned}
& \widehat{A} \Pi^{T}=\left[\begin{array}{ccccc}
U_{1}\left[\begin{array}{c}
\Lambda_{1} \\
0
\end{array}\right] & 0 & 0 & 0 & 0 \\
0 & 0_{n-n_{1}} & \bar{A}_{21} & U_{2}\left[\begin{array}{c}
\Lambda_{2} \\
0
\end{array}\right] & 0
\end{array}\right] \times \\
& \times\left[\begin{array}{cccc}
I_{n_{1}} & 0 & 0 & 0 \\
0 & 0 & I_{n-n_{1}} & 0 \\
0 & I_{n_{1}+n_{2}} & 0 & 0 \\
0 & 0 & 0 & I_{n-\left(n_{1}+n_{2}\right)}
\end{array}\right] \\
& =\left[\begin{array}{ll}
C & 0_{L \times\left(2 n-2 n_{1}-n_{2}\right)}
\end{array}\right]
\end{aligned}
$$

Further, from (A.28)

$$
\Pi \widehat{F} \Pi^{T}=\left[\begin{array}{cc}
I-\widehat{\Upsilon} & 0 \\
\times_{1} & \times_{2}
\end{array}\right]
$$

where $\left[\begin{array}{ll}\times_{1} & \times_{2}\end{array}\right]$ has $2\left(n-n_{1}\right)-n_{2}$ rows and

$$
\widehat{\Upsilon}=\left[\begin{array}{ccc}
\frac{\Lambda_{1}^{2}}{\alpha} & -I_{n_{1}}+\frac{\Lambda_{1}^{2}+2 \bar{A}_{12}^{T} \bar{A}_{21}}{\alpha} & \frac{2 B}{\alpha} \\
\frac{2 \Lambda_{1}^{2}}{\alpha} & \frac{2 \Lambda_{1}^{2}+4 \bar{A}_{21}^{T} \bar{A}_{21}}{\alpha} & \frac{4 B}{\alpha} \\
0 & \frac{4 B^{H}}{\alpha} & \frac{4 \Lambda_{2}^{2}}{\alpha}
\end{array}\right]
$$

Then the result follows.

Lemma A.5. Under the conditions of Lemma A.1, $A(z I-F)^{-1}$ has no poles at 1. 
Proof. Because of Lemmas A.2 and A.4, It suffices to show that $\Upsilon$ defined in Lemma A.4 has no eigenvalues at 1. Choose

$$
T=\left[\begin{array}{ccc}
I_{n_{1}} & -I_{n_{1}} / 2 & 0 \\
0 & I_{n_{1}} & 0 \\
0 & 0 & I_{n_{2}}
\end{array}\right]
$$

and note that

$$
T^{-1}=\left[\begin{array}{ccc}
I_{n_{1}} & I_{n_{1}} / 2 & 0 \\
0 & I_{n_{1}} & 0 \\
0 & 0 & I_{n_{2}}
\end{array}\right]
$$

Then

$$
\begin{aligned}
& T \Upsilon T^{-1}= \\
& =\left[\begin{array}{ccc}
I_{n_{1}} & I_{n_{1}} / 2 & 0 \\
-\frac{2 \Lambda_{1}^{2}}{\alpha} & I_{n_{1}}-\frac{2 \Lambda_{1}^{2}+4 \bar{A}_{21}^{T} \bar{A}_{21}}{\alpha} & -\frac{4 B}{\alpha} \\
0 & -\frac{4 B^{H}}{\alpha} & I-\frac{4 \Lambda_{2}^{2}}{\alpha} \\
I_{n_{1}} & I_{n_{1}} & 0 \\
-\frac{2 \Lambda_{1}^{2}}{\alpha} & I_{n_{1}}-\frac{3 \Lambda_{1}^{2}+4 \bar{A}_{21}^{T} \bar{A}_{21}}{\alpha} & -4 B \\
0 & -4 B^{H} & I-\frac{4 \Lambda_{2}^{2}}{\alpha}
\end{array}\right] T^{-1}
\end{aligned}
$$

Now, $T \Upsilon T^{-1}$ has an eigenvalue at 1 iff there exists

$$
\eta=\left[\begin{array}{l}
\eta_{1} \\
\eta_{2} \\
\eta_{3}
\end{array}\right] \neq 0
$$

such that

$$
\left[\begin{array}{ccc}
0 & -I_{n_{1}} & 0 \\
\frac{2 \Lambda_{1}^{2}}{\alpha} & \frac{3 \Lambda_{1}^{2}+4 \bar{A}_{21}^{T} \bar{A}_{21}}{\alpha} & \frac{4 B}{\alpha} \\
0 & \frac{4 B^{H}}{\alpha} & I-\frac{4 \Lambda_{2}^{2}}{\alpha}
\end{array}\right]\left[\begin{array}{l}
\eta_{1} \\
\eta_{2} \\
\eta_{3}
\end{array}\right]=0 .
$$

Now the first block equation implies $\eta_{2}=0$. Further because of (A.30), the third block equation gives $\eta_{3}=0$. Thus as $\Lambda_{1}>0$, the second block equation assures that $\eta_{1}=0$, leading to a contradiction.

Thus Lemma 4.1 follows from Lemmas A.1 and A.5.

\section{REFERENCES}

[1] J. BAILlieul AND A. SURI, Information patterns and hedging brockett's theorem in controlling vehicle formations, in Proceedings of CDC, Maui, Hawaii, December 2003.

[2] T. Balch And R. C. ARkin, Behavior-based formation control for multirobot teams, IEEE Transactions on Robotics and Automation,, pp, 926 - 939, December 1998. 
[3] D. W. Clarke and P. J. Gawthrop, Self-tuning control, Proceedings of the Institution of Electrical Engineers, pp. 633-640, 1979

[4] W. B. Dunbar and R. M. Murray, Distributed receding horizon control with application to multi-vehicle formation stabilization, Automatica, 42(2006), pp. 549-558.

[5] T. Eren, W. Whiteley, B.D.O. Anderson, A.S. Morse, And P. N. Belhumeur, Information structures to secure control of rigid formations with leader-follower architecture, in Proceedings of JACC, June 2005.

[6] T. Eren, W. Whiteley, A.S. Morse, P.N. Belhumeur, and B.D.O. Anderson, Sensor and network topologies of formations with direction, bearing, and angle information between agents, in Proceedings. 42nd IEEE Conference on Decision and Control , pp. 3064-3069, December, 2003.

[7] J. A, FAX And R. M. Murray, Graph laplacians and stabilization of vehicle formations, in Proceedings of 15th IFAC World Congress, July 2002, Barcelona, Spain.

[8] — Information flow and cooperative control of vehicle formations, Transactions on Automatic Control, pp. 1465-1476, 2004.

[9] E. Franco, T. Parisini, and M. M. Polycarpou, Cooperative control of discrete-time agents with delayed information exchange: A receding-horizon approach, in Proceedings of 43rd IEEE Conference onDecision and Control, pp. 4274-4279, December 2004.

[10] S. S. Ge And C. H. FuA, Queues and artificial potential trenches for multirobot formations, IEEE Transactions on Robotics, 21:4(2005), pp. 646-656.

[11] S. Glavski, M. Chaves, R. Day, P. NaG, A. Williams, and W. Zhang, Vehicle networks: Achieving regular formation, in American Control Conference, June 2003.

[12] A. Jadbabaie, J. Lin, AND A. S. Morse, Coordination of groups of mobile autonomous agents using nearest neighbor rules, IEEE Trabsactions on Automatic Control, pp. 988 - 1001, June 2003.

[13] C. R., Johnson, JR. And E. TsE, Adaptive implementation of one-step-ahead optimal control via input matching, IEEE Transactions on Automatic Control, pp. 865-872, 1978.

[14] M. E. Khatir And E. J. Davison, Bounded stability and eventual string stability of a large platoon of vehicles using non-identical controllers, in Proceedings of JACC, 2004.

[15] T. Lefebvre, H. Bruyninckx, And J. De Schutter, Polyhedral contact formation identification for autonomous compliant motion: Exact nonlinear bayesian filtering, IEEE Transactions on Robotics, 21:1(2005), pp. 124.

[16] J. Lin, A. Stephen Morse, and Brian D.O. Anderson, The multi-agent rendezvous problem -part 1: The synchronous case, SIAM Journel on Control and Optimization, 2006.

[17] L. Moreau, Stability of multiagent systems with time-dependent communication links, IEEE Transactions on Automatic Control, 50:2(2005), pp. 169-182.

[18] N. Motee And A. JadBABAie, Receding horizon control of spatially distributed systems over arbitrary graphs, in 45th IEEE Conference on Proceedings of Decision and Control, 2006 pp. 3467-3472.

[19] R. Olfati-Saber and R. M. Murray, Consensus problems in networks of agents with switching topology and time-delays, IEEE Transactions on Automatic Control, 49:9(2004), pp. $1520-1533$.

[20] R. Olfati- Saber, W.B. Dunbar, and R.M. Murray, Cooperative control of multi-vehicle systems using cost graphs and optimization, in Proveedings of JACC, pp. 2217-2222, 2003.

[21] R. Sepulchre, D. Paley, and N. Leonard, Collective motion and oscillator synchronization, in Proceedings of the Block Island Workshop on Cooperative Control, June 2003.

[22] D. Swaroop And J. K. Hedrick, String stability of interconnected systems, IEEE Transactions on Automatic Control, 41:3(1996), pp. 349-357.

[23] P. Tabuada,G. J. Pappas, and P. Lima, Motion feasibility of multi-agent formations, IEEE TRANSACTIONS ON ROBOTICS, 21:3(2005), pp. 387-392. 
[24] J. Toner And Y. Tu, Flocks, herds, and schools: A quantitative theory of flocking, Physical Review E, 58:4(1998), pp. 4828-4858.

[25] T. Vicsek, A. Czirok, E. Ben-Jacob, I. Cohen, and O. Shochet, Novel type of phase transition in a system of self-driven particles, The American Physical Society, 75:6(1995), pp. $1226-1229$.

[26] A. Williams, S. Glavaski, and T. Samad, Formations of formations: Hierarchy and stability, in Proceedings of JACC, June 2004.

[27] H. Yamaguchi and G. Beni, Distributed autonomous formation control of mobile robot groups by swarm-based pattern generation, in Proc. of the 2nd Int. Symp. on Distributed Autonomous Robotic Systems (DARS 96), 141-145, 1996.

[28] J. YAN AND R.R. Bitmead, Coordinated control and information architecture, in Proceedings of 42nd IEEE Conference on Decision and Control, pp. 3919-3923, 2003.

[29] C. Yu, B. D. O. Anderson, S. Dasgupta, and B. Fidan, Control of minimally persistent formations in the plane, SIAM J. Control Optim., 48(2009) , pp. 206-233.

[30] F. Zhang, A. O'Connor, D. Luebke, and P. S. Krishnaprasad, Experimental study of curvature-based control laws for obstacle avoidance, in Proceedings of IEEE International Conference on Robotics and Automation, April 2004. 